Geopolítica(s) Revista de estudios sobre espacio y poder ISSN: 2172-3958

https://dx.doi.org/10.5209/geop.63962

\title{
Geografía de la participación electoral y diferenciación socioespacial en Ciudad Juárez, Chihuahua (México)
}

\author{
Vladimir Hernández Hernández y Liliana De Haro De León ${ }^{2}$
}

Recibido: 3 de abril de 2019 / Aceptado: 2 de marzo de 2020

Resumen. Recientemente los estudios de geografía electoral en México están incorporando conceptos como dependencia espacial para valorar la dimensión geográfica como una de las dimensiones explicativas del voto. El artículo plantea como objeto de estudio la relación entre la diferenciación socioespacial y la participación ciudadana en la formación de núcleos entre las secciones electorales de Ciudad Juárez, México. En esta dependencia se toma en cuenta la importancia de los efectos espaciales, dados estos últimos por la proximidad de las secciones electorales. Este trabajo presenta técnicas de análisis espacial (autocorrelación espacial, regresiones espaciales y métodos cartográficos). Entre los principales resultados se encontró evidencia de agrupamientos espaciales, lo que significa que en el espacio geográfico los valores del porcentaje participación electoral y de diferenciación socio espacial tiene una mayor relación con aquellos espacios más cercanos que los distantes, donde una disminución en el nivel de diferenciación socioespacial aumenta el nivel de participación electoral. Y estos valores varían en el espacio urbano verificando que la configuración espacial de la participación ciudadana en Ciudad Juárez durante las elecciones presidenciales del 2012 no se produce de manera aleatoria quedando evidencia de la interdependencia y retroalimentación entre las secciones electorales ratificando el efecto de vecindario.

Palabras clave: geografía electoral; secciones electorales; análisis espacial; regresión espacial; Ciudad Juárez.

\section{[en] Geography of Electoral Participation and Socio-spatial Differentiation in Ciudad Juárez, Chihuahua, Mexico}

\begin{abstract}
Studies of electoral geography in Mexico have recently incorporated concepts such as spatial dependence. Thereby, it is sought underscore the relevance of the geographic dimension as one of the explanatory dimensions of voting. The article investigates the relationship between sociospatial differentiation and citizen participation in the formation of nuclei between the electoral sections of Ciudad Juárez, Mexico. In exploring this, the importance of spatial effects resulting from the proximity of electoral sections are considered. This work presents spatial analysis techniques (spatial autocorrelation, spatial regressions and cartographic methods). Among the main results, evidence of spatial groupings was found, which means that in the geographical space the values of the percentage of electoral participation and socio-spatial differentiation have a greater relationship with those spaces
\end{abstract}

1 Profesor e investigador. Universidad Autónoma de Ciudad Juárez.

E-mail: vladimir.hernandez@uacj.mx

2 Investigadora independiente. Doctora en Geografía y Ordenación Territorial

E-mail: lilydeharo@hotmail.com 
that are closer than the distant ones. A decrease in the level of socio-spatial differentiation increases the level of electoral participation. These values vary across the urban space verifying that the spatial configuration of citizen participation in Ciudad Juárez during the 2012 presidential elections does not occur randomly. In short, the article provides evidence of the interdependence and feedback between the electoral sections which, in turn, ratifies the neighbourhood effect.

Keywords: electoral geography; electoral sections; spatial analysis; spatial regression; Ciudad Juárez.

\section{[pt] Geografia da participação eleitoral e diferenciação socioespacial em} Ciudad Juárez, Chihuahua, México

Resumo. Recentemente, os estudos de geografia eleitoral no México estão incorporando conceitos como dependência espacial para valorizar a dimensão geográfica como uma das dimensões explicativas do voto. $\mathrm{O}$ artigo tem como objeto de estudo a relação entre diferenciação socioespacial e participação cidadã na formação de núcleos entre as seções eleitorais de Ciudad Juárez, México. Essa dependência leva em consideração a importância dos efeitos espaciais, especialmente a proximidade das seções eleitorais. Apresentam-se técnicas de análise espacial (autocorrelação espacial, regressões espaciais e métodos cartográficos). Dentre os principais resultados, foram encontradas evidências de agrupamentos espaciais, o que significa que no espaço geográfico os valores da porcentagem de participação eleitoral e da diferenciação socioespacial têm uma relação maior com os espaços mais próximos que os distantes. Uma diminuição no nível de diferenciação socioespacial aumenta o nível de participação eleitoral. E esses valores variam no espaço urbano, verificando que a configuração espacial da participação do cidadão em Ciudad Juárez durante as eleições presidenciais de 2012 não ocorre aleatoriamente, deixando evidências de interdependência e feedback entre as seções eleitorais, fato que ratifica o efeito de vizinhança.

Palavras-chave: geografia eleitoral; seções eleitorais; análise espacial; regressão espacial; Ciudad Juárez.

Sumario. Introducción. 1. Las concepciones teóricas de los estudios electorales. 1.1. Geografía electoral. 1.2. Segregación socioespacial. 2. Ciudad Juárez como caso de estudio. 3. Metodología y bases de datos. 3.1. 3.1. Elaboración de los índices. 4. Resultados y análisis. 4.1. Indicadores de autocorrelación espacial. 4.2. Análisis de regresión. Conclusiones. Referencias.

Cómo citar: Hernández Hernández, V., y De Haro De León, L. (2020). Geografía de la participación electoral y diferenciación socioespacial en Ciudad Juárez, Chihuahua (México). Geopolítica(s). Revista de estudios sobre espacio y poder, 11(1), 145-172.

\section{Introducción}

El resultado de las elecciones no es neutral respecto al espacio geográfico. En la actualidad, la afirmación con la que iniciamos este trabajo es cada vez más aceptada en el medio académico derivado de las evidencias empíricas de diferentes contextos, en una gama de diferentes mecanismos y una diversidad de escalas. Cutts y Weber (2010) menciona que votar es una actividad aprendida, resultado de la interacción de las personas con su entorno material, redes sociales o través de campañas políticas muy particulares, y todo sucede en un lugar; por lo tanto, no hay sorpresa en afirmar que existen variaciones espaciales en los patrones de votación. Weichelt (2018, p.17), en su trabajo sobre el voto hispano en Estados Unidos, remarca que la información de los patrones espaciales es importante para las campañas electorales; el autor hace referencia a la estrategia empleada por el grupo del candidato Barak 
Obama, y plantea la hipótesis que la comprensión de los patrones de votos de las elecciones previas, sin duda, marcaron una diferencia en el resultado de las elecciones presidenciales del 2008.

Lo anterior es una breve referencia a los trabajos, que no son pocos, que afirman que los resultados de las elecciones no son independientes del espacio. Sobre este tema se pueden encontrar una amplia variedad de investigaciones que de forma explícita buscar analizar los agrupamientos y explicaciones de los resultados electorales (O'Loughlin et al., 1998; O'Loughlin, 2002, 2003; Darmofal, 2003; Tam Cho y Nicley, 2008; Klos, 2008; Soares y Terron, 2008; Chen y Rodden, 2009; Seabrook, 2009; Lefebvre y Robin, 2009; Rodden 2010; Crespin, Darmofal y Eaves, 2011).

En México encontramos los trabajos de Vilalta (2004, 2006, 2007, 2008), y más tarde los de Lizama (2012), Hernández (2015), Ávila-Eggleton y GutiérrezSánchez (2017) y De Haro (2018b). En el contexto nacional las escalas de análisis han sido a nivel de entidad o estado federal, municipal y distritos electorales. Es Vilalta $(2008$, p.572) quien recurre a la estimación de modelos de regresiones espaciales, específicamente de rezago espacial, para analizar que en la Ciudad de México los bajos niveles de marginación y la alta segregación socioeconómica presenta una marcada geografía del voto y que esta se halla más dividida geográficamente en su voto que en su ingreso.

En el contexto local se cuenta con los antecedentes de los trabajos de Hernández y De Haro (2017) y De Haro (2018b). Los primeros realizaron un análisis geoespacial de la elección para gobernador en el estado de Chihuahua durante las elecciones estatales del año 2010. El trabajo en cuestión se enfocó en la estimación de la dependencia espacial a escala municipal. Y una de las conclusiones indica que la participación electoral es menor mientras más urbanizado sea el municipio. El segundo abordó el tema del abstencionismo mediante técnicas de análisis exploratorio de datos espaciales para las elecciones del estado de Chihuahua del período comprendido entre los años del 2004 al 2016. En cuanto a los trabajos de segregación socioespacial en Ciudad Juárez, la revisión de la literatura coincide en describir la fragmentación urbana resultado de la dinámica económica y la inserción de la ciudad en la división internacional del trabajo; en términos generales, es una sociedad urbana altamente polarizada social y económicamente (Fuentes, 2009; Fuentes y Hernández, 2013; López y Peña, 2017; Fuentes, Peña y Hernández, 2018).

En este trabajo, se propone como tema de investigación la relación entre la diferenciación socioespacial y la participación ciudadana en la formación de núcleos entre las secciones electorales de Ciudad Juárez; en este vínculo se toma en cuenta la influencia de los efectos espaciales como resultado de la proximidad entre las secciones electorales. De tal forma que, una vez analizada de manera comparada la agrupación de las dos dimensiones del estudio, se considera la asociación mediante la vecindad geográfica. La pregunta central del trabajo a la que se pretende dar respuesta es la siguiente: ¿Cuál es el papel de la dependencia espacial en la participación electoral propiciada por la desigualdad socioespacial en las secciones electorales de Ciudad Juárez, Chihuahua? Y la hipótesis asociada se plantea a continuación: los efectos espaciales, a través de la contigüidad y la diferenciación socioespacial condicionan la participación electoral en Ciudad Juárez, mostrando efectos no estacionarios en la ciudad. 
Además de la presente introducción, el estudio se ha ordenado en cuatro secciones. La primera se divide en dos apartados: por una parte, hacemos una revisión sobre los estudios de geografía electoral, y luego nos centramos en la revisión de la segregación socioespacial. La segunda sección describe las características del estudio de caso: Ciudad Juárez. La tercera detalla la metodología del estudio. Y la cuarta muestra los resultados del análisis, los cuales presentan los cálculos de la autocorrelación global y local, la información cartográfica de los indicadores y se cierra con la estimación de los modelos de regresión espacial y geográficamente ponderada. Al final se incluyen las conclusiones de este trabajo.

\section{Las concepciones teóricas de los estudios electorales}

Los estudios electorales son un campo interdisciplinario que enlaza áreas del conocimiento como el derecho, la sociología, la ciencia política y la geografía. La postura teórica de este trabajo se asume a partir del enfoque de la geografía electoral. Sin embargo, a continuación, se realiza una breve revisión de otros enfoques pertinentes en el estudio de los fenómenos electorales. En primer lugar, la denominada teoría sociológica del voto asume que el votante actúa en la política como es en la sociedad (Ichuta, 2017, p.89); los estudiosos desde esta postura examinan con atención las principales dimensiones sociológicas como son la raza, la ocupación, la clase social y la religión (Johnston y Pattie, 2006; Mohd, Azlina y Mohd, 2016; Maggini, 2017). A continuación, la teoría psicológica del voto propone que la elección de los votantes está influenciada por las creencias y sensaciones que los individuos tienen acerca de los partidos políticos (Harder y Krosnick, 2008; LewisBeck, Jacoby, Norpoth y Weisberg, 2008). El tercer enfoque denominado de la elección racional plantea que la elección del votante es resultado de la evaluación de las ofertas de los partidos en función de sus demandas, sintetizándose en la elección de un determinado partido político a través de un cálculo costo-beneficio (McKelvey y Ordeshook, 1986; Aldrich, 1993; Riker, 1997). Sobre este planteamiento se definió la teoría racional del voto. Finalmente, la teoría de la elección pública que sintetiza en sus propuestas los aportes de cada uno de los enfoques previamente expuestos mediante la puesta en práctica de complejos modelos estadísticos (Box-Steffensmeier, Brady y Collier, 2008; Hedström, 2008; Morton y Williams, 2008).

\subsection{Geografía electoral}

La geografía electoral ha sido considerada tradicionalmente como una subdisciplina de la geografía política. Es posible identificar el arranque de este enfoque con el inicio del siglo XX, por lo tanto, goza de una larga historia intelectual (Weichelt, 2018, p.12). Uno de los trabajos académicos fundadores es atribuido a Siegfried ${ }^{3}$ (2010) quien intentó explicar que la dispersión de la población y el latifundio versus la concentración poblacional y la pequeña burguesía influyen en la elección de

La primera edición del trabajo de André Siegfried se publicó en 1913 con el título Tableau politique de la

France de l'ouest sous la troisième république: 102 cartes et croquis, 1 carte hors texte. 
una alternativa electoral; por la derecha en el primer caso y por la izquierda en el segundo. A este autor se le atribuyen un importante rol en el surgimiento de la geografía electoral. La primera mitad del siglo XX se caracterizó por un énfasis de la geografía electoral con una perspectiva funcionalista. Agnew (1990) a partir de la década de 1960 divide en cuatro grandes etapas el moderno pensamiento de la geografía electoral. El primero de ellos lo denomino período de modernizaciónnacionalización (1965-1972) que básicamente se enfocaba en el análisis de los flujos de información y la integración económica argumentando una homogeneización espacial — nacional — del comportamiento electoral. La segunda etapa definida como del bienestar demográfico, la premisa de esta etapa se puede resumir mediante la relación desigual entre la distribución y acceso a bienes y servicios públicos que a grandes rasgos definiría la implicación de los diversos grupos en los procesos político. Los aportes de Johnston (1979); Taylor y Johnston (1979) enfatizan las explicaciones espaciales de los comportamientos políticos y las consecuencias de los arreglos políticos en la distribución espacial de los beneficios sociales. Agrupan el interés de estudio en tres grandes enfoques: el primero lo denominan geografía del voto, y tratan de comparar las distribuciones del voto con la distribución de otro fenómeno; el segundo lo constituyen los estudios de las influencias geográficas en las votaciones - por ejemplo, los flujos de información-, y el tercer foco de interés se refiere a los efectos de la división territorial — geografía de la representación - en circunscripciones electorales.

La tercera etapa denominada desarrollo desigual se avoca a señalar las diferencias entre los centros y las periferias del poder. En Estados Unidos las oposiciones políticas entre republicanos y demócratas y los trabajos de Johnston y Pattie (2006) en el caso de Gran Bretaña. Este período analiza los conflictos entre las regiones y el poder central - $\mathrm{o}$ federal - El cuarto período, definido como perspectiva microsociológica, a partir de la década de los 1980 enfatiza el "lugar" como base para explicar el comportamiento político. Fue durante este periodo que Johnston, Gregory y Smith (1987) definieron las áreas de estudios de la geografía electoral, que incluyen, a modo de resumen: 1) la organización espacial de las elecciones, 2) las variaciones espaciales en las pautas de los votos, 3) la influencia de los factores espaciales en las decisiones sobre el voto, 4) las estructuras espaciales de representación en organismos legislativos y 5) las diferencias en la partición geográfica del poder.

Johnston y Pattie (2003) mencionan que la geografía electoral se centra en la interacción del espacio, el lugar y los procesos electorales. Para estos autores el análisis se centra en los impactos del contexto geográfico - los lugares - en las decisiones de los votantes y el funcionamiento de los sistemas electorales. Plantean que la disciplina es un puente entre la geografía humana y la ciencia política. En países como Francia, Inglaterra, Estados Unidos desde hace más de cincuenta años se ha venido estudiando el comportamiento electoral con énfasis en el análisis espacial. Recientemente se sugiere utilizar fuentes de información de pequeña escala para lograr una mejor interpretación de los resultados en cuanto a las conductas individuales en áreas urbanas, abstencionistas, lugares inseguros y peligrosos, así como, zonas de migrantes, conectando las diferencias regionales y locales (Beaugutte y Colange, 2013).

Finalmente, a partir del trabajo de Agnew (2002) se definieron los tres aspectos fundamentales del enfoque del "lugar": un lugar es 1) el sitio donde la mayoría de 
las personas realiza su vida cotidiana; 2) es además una ubicación vinculada a través de distintas redes a una amplia gama de otras escalas regionales o nacionales; y 3 ) es un sitio con el que las personas se identifican. Si bien, en un primer momento cada uno de los tres aspectos previamente mencionados se consideran por separado, estos están estrechamente interrelacionados y desde la perspectiva de Agnew (2002) y Johnston y Pattie $(2003,2006)$ tienen un papel fundamental en la creación de actitudes políticas. Por lo tanto, la perspectiva del lugar se considera un relevante argumento teórico multiescalar en la creación de identidades políticas personales y la movilización de apoyo a los partidos políticos (Johnston y Pattie, 2006, p.43).

En México, a diferencia de otros contextos, los primeros trabajos se remontan a la década de 1970 enmarcados dentro de la teoría de la modernidad con el propósito de encontrar explicaciones sólidas a los patrones de votación de acuerdo con la dualidad rural/urbana. Por lo que se refiere a las definiciones, el debate es amplio entre la adscripción de la geografía electoral como una rama de la geografía política resultado del paradigma cuantitativo (Monzón, 2009), mientras que para otros la geografía electoral tiene como principal interés la demarcación o división de las funciones electorales que se han establecido en el país (Guerrero, 2001). También se ha mencionado que es ámbito de estudio todo el proceso electoral desde las campañas, la publicidad hasta el análisis de los resultados.

No existe una delimitación evidente en la evolución de la geografía electoral mexicana. Las tradiciones corológica y ecológica continúan como enfoques predominantes $\mathrm{y}$, en menor medida, se están incorporando a la discusión trabajos empíricos a partir de la identificación de dependencias espaciales (Hernández y De Haro, 2017; De Haro, 2018b; Charles-Leija, Torres y Colima, 2018). Aun así, la tradición de los estudios electorales en México está polarizada entre aquellos que niegan los aportes de la geografía a los estudios electorales, reduciéndolos a un elemento descriptivo (Emmerich, 1993; Molinar, 1991), y, por otra parte, quienes argumentan que la dimensión espacial es un factor influyente en el comportamiento electoral (Peschard, 2008; Vilalta, 2008; Gómez-Tagle, 2009). Un trabajo interesante que resumen parte de la tradición de los estudios del comportamiento electoral en México es la obra de Ichuta (2017).

\subsection{Segregación socioespacial}

La segregación ha estado vinculada desde su conceptualización como tema de interés académico con el estudio de la ciudad. Debido a su larga trayectoria como fenómeno urbano el concepto se ha definido de diversas maneras; sin embargo, se pueden identificar dos grandes grupos de enunciaciones. En primer lugar, la de mayor generalización se refiere a la forma en cómo se organiza la ciudad; por otra parte, las definiciones particulares hacen alusión a la diferenciación en el espacio geográfico debido a fenómenos de tipo económico, de raza, políticos y sociales, entre otros (Pérez-Campuzano, 2011, p.405). Al final, la definición más habitual se refiere a la separación de los grupos en el espacio.

La segregación como fenómeno sustenta su desarrollo a partir de dos enfoques: 1) Desde su dimensión geográfica, hace alusión al postulado de dependencia espacial de la siguiente manera, es decir, los grupos sociales se aglomeran en algunas zonas de las ciudades, principalmente como resultado de sus condiciones económicas y en menor medida de otro tipo de características, y, por lo tanto, las zonas 
ocupadas tienen mayor homogeneidad al interior que las zonas alejadas. 2) En segundo lugar, a partir de la mirada de la sociología urbana, el estudio de la segregación se centra en las causas y su expresión en los grupos más vulnerables de la ciudad (Fuentes y Hernández, 2013). En este sentido desde la perspectiva geográfica una de las categorías que más se ha trabajado para comprobar la división social del espacio asociado a los patrones de localización intraurbana es la segregación socioespacial (Alegría, 1994; Vilalta, 2007).

Es muy interesante que al revisar la extensa literatura del tema pocas veces se menciona que la vida en las ciudades impone por sí misma divisiones que se traducen en espacios diferenciados. En este sentido, Pérez-Campuzano (2011, p.407) menciona que la segregación es un tema de acceso y poder en distintas esferas de la vida y su manifestación espacial, es precisamente, la expresión de las oportunidades y de poder con el que los grupos cuenten; en consecuencia, la organización espacial es el resultado de la capacidad de decidir y del poder para llevarlas a cabo. Lo anterior es relevante para el propósito de este trabajo, porque el espacio es "arena y receptor" de los conflictos. Otro autor expresa que la segregación es la manifestación de la división social del espacio urbano, es decir, que el lugar de residencia de un individuo lo diferencia de otros individuos, creando con ello una distancia física y psicológica. Y concluye que la existencia de la separación es resultado del ejercicio del poder y de la voluntad de separación de los grupos (Madoré, 2004). En cambio, Jargowsky y Kim (2005) explican que el espacio inmediato -el barrio- de los individuos es un escenario de interacciones que influyen en las actitudes y aspiraciones de quienes lo habitan. Finalmente, la segregación es un fenómeno multidimensional que no es fácil definir y menos valorar, donde es relevante identificar las formas de interacción y las escales espaciales y temporales donde se manifiesta (Massey y Denton, 1988; Massey, 2012).

Al respecto, Massey y Denton (1988) mencionan que la segregación residencial a nivel general es un tema complejo. Ellos mencionan que los grupos sociales pueden vivir separados unos de otros y ser segregados de varias maneras. Por ejemplo, grupos minoritarios pueden estar sobrerepresentados en algunas áreas y subrepresentados en otras; es decir, con una uniformidad variable. En consecuencia la exposición a los miembros mayoritarios está limitada en virtud de que rara vez comparten un barrio con ellos. Asimismo, pueden concentrarse espacialmente en una área pequeña, ocupando menos espacio físico que los miembros mayoritarios o pueden estar centralizados espacialmente, congregándose alrededor del núcleo urbano y ocupando una ubicación más central que la mayoría. Las zonas de minorías pueden agruparse fuertemente para formar un enclave contiguo grande o estar disperso alrededor del área urbana. En consecuencia, está complejidad tiene sus efectos en las mediciones y en las unidades de análisis.

En el contexto nacional se hace referencia a lo mencionado por Alegría (1994) quien propone una conceptualización diferente sobre la segregación, definiéndola como el alejamiento o vecindad geográfica entre los grupos sociales y no de estos con relación a cualquier otro elemento del entorno urbano valorado por la accesibilidad. Para este autor son dos dimensiones las que manifiestan el hecho: 1) en primer lugar, la capacidad con que cada persona entra a la disputa por los recursos urbanos, lo que dependerá de 2) la segunda cualidad, el grado y clase de recursos económicos y de conocimiento con lo que cuente. Es así como la localización en el espacio urbano obedecerá a ambos tipos de cualidades. Esta clasificación de grupos 
sociales permitirá conocer las relaciones de poder entre las personas, instituciones y otras organizaciones locales, a la par de su estructura en la distribución del capital específico del mismo lugar. Aquí coincide con lo expresado por PérezCampuzano (2011).

En México y en ciudades como la analizada en este trabajo es muy importante considerar que la oferta de la vivienda formal repercute en el acomodo de los diversos grupos sociales, porque divide el mercado conforme al ingreso, el gusto y preferencias de los clientes, elaborando versiones similares de una misma vivienda que se refleja en el precio final del bien (López y Peña, 2017). De tal forma, el análisis de la segregación depende de las condiciones sociales de los individuos. En ocasiones, las características sociodemográficas pueden ser establecidas a partir de la medición de la accesibilidad urbana en conjunto con las particularidades de la vivienda; además, la definición sociodemográfica, también se valora con relación a los bienes de consumo que tienen las viviendas, esto es particularmente útil, cuando los datos son escasos. En este sentido una definición de la segregación urbana se refiere a la divergencia en las condiciones de acceso de la población a los bienes de una ciudad, resultado de su localización en el espacio urbano. La segregación es obra del mercado y segmentación formal e informal de la vivienda y de su interdependencia con las bases sociales y que a su vez se trascribe territorialmente en la separación de las clases sociales en el territorio (Pérez-Tamayo, Gil-Alonso y Bayona-i-Carrasco, 2017). Así mismo Vilalta (2008), propone que desde la teoría de la globalización se puede explicar la distribución del ingreso, el aumento en diferencias salariales entre trabajadores calificados y no calificados, el incremento de mujeres que participan en las actividades económicas y, en consecuencia, los aumentos en la segregación socioespacial.

Una de las principales preocupaciones de los estudiosos de la segregación es su medición. En la actualidad derivado de los avances de la computación y en la mayor disponibilidad de datos se avanzó considerablemente en este campo (PérezCampuzano, 2011). Existe un consenso en señalar a los trabajos de Massey y Denton (1988) y Massey (2012) como el soporte teórico de las mediciones de la segregación, en la que ellos reconocieron cinco elementos: desigualdad, exposición, concentración, centralización y agrupamientos. Sin embargo, los autores previamente citados coinciden en señalar que la mayoría de los índices remiten al índice de disimilitud de Duncan y Duncan (1955) que sirvió como medida estándar de segregación residencial, sobre todo para medir la segregación espacial entre los grupos sociales. Es importante mencionar que una de las principales críticas metodológicas realizadas al índice de disimilitud es su naturaleza no-espacial; por lo que diversos autores (Wong, 2003; Reardon y O'Sullivan, 2004) propusieron incorporar una matriz de contigüidad u otras definiciones que consideraran explícitamente la naturaleza espacial del fenómeno.

\section{Ciudad Juárez como caso de estudio}

Ciudad Juárez se sitúa en la frontera norte de México; su límite territorial al sur de El Paso, Texas, en Estados Unidos propicia una dinámica de flujo migratorio fronterizo muy importante. ha presentado dinámicas de población constante desde el año de 1960 a la fecha. Por ejemplo, en 1940 la población en la ciudad se contabi- 
lizó en cerca de 48,000 habitantes, y el más reciente conteo de población en 2015 registró un total de 1,300,000 habitantes (INEGI, 2019).

La composición socioespacial se rige por el dominio del mercado global en la regularización del suelo, donde las características de los grupos sociales en algunas zonas y su homogeneidad se relaciona a intereses propios de los individuos. El acceso a la vivienda formal se puede clasificar conforme a los niveles de ingresos en: altos, medios o bajos (López y Peña, 2017). Durante las últimas décadas la ciudad se caracterizó por una fuerte oferta de vivienda de interés social: entre los años de 2001 al 2006 se construyeron aproximadamente 97,000 viviendas de interés social, lo que representó el 86.8 por ciento de total de la vivienda construida en la ciudad en el mismo período (Fuentes y Hernández, 2014, p.372). Se cuenta con una población muy amplia del sector industrial, a diferencia de otros municipios no fronterizos en el estado; pero aunado a lo anterior, en la actualidad se registra una alta desocupación de dichas viviendas (Fuentes y Hernández, 2014). Los procesos anteriores han dibujado una estructura urbana caracterizada por una expansión constante y una marcada polarización socioterritorial.

Otra de las dimensiones relevantes para este trabajo son las condiciones de la pobreza urbana, si bien no es éste el espacio para realizar una discusión amplia sobre el tema, se deben considerar las condiciones de vida de la población en tres aspectos: a) bienestar económico, que impacta a las necesidades asociadas a los bienes y servicios que se adquieren mediante el ingreso; b) la dimensión social, que se encuentran en el ejercicio de los derechos para el desarrollo social; y c) el contexto territorial, que incorpora un aspecto que trasciende en el ámbito individual, en tanto, se refiere a las características geográficas, sociales, culturales y aquellas asociadas al grado de cohesión social y grado de accesibilidad para el desarrollo social. En este sentido, Fuentes, Peña y Hernández (2018, p.12) estimaron que los habitantes que vivían en situación de pobreza en la ciudad en 2012 eran un 39.8 de la población total y en pobreza extrema el 5.6 por ciento. En cambio, el 22.3 por ciento de la población no registró ninguna dimensión de carencia y privación social.

La historia reciente de Ciudad Juárez ha estado marcada, lamentablemente, por la violencia e inseguridad. No son situaciones exclusivas de esta ciudad, pero las manifestaciones son complejas y sobresalen los feminicidios y las ejecuciones ligadas al narcotráfico. Los tres niveles de gobierno (local, estatal y federal) construyeron iniciativas para detener los valores atípicos de violencia mediante proyectos de mejoramiento de las condiciones sociales y el aumento de oportunidades de desarrollo para las personas (Cervera y Monárrez, 2013). Si bien no es objetivo de este trabajo valorar la participación en las elecciones y las condiciones de violencia e inseguridad, se considera que son situaciones singulares del "lugar".

Finalmente, realizaremos unas breves notas sobre la institucionalidad democrática en Ciudad Juárez. Es ilustrativo como Borunda (2008, pp.100-103) compara la designación de candidatos a nivel nacional y estatal con los candidatos locales, los alcaldes, pero estos últimos con matices relacionados con las élites empresariales y, en el caso de Ciudad Juárez, con la influencia religiosa. A continuación, este autor hace referencia al caso emblemático de Ciudad Juárez y otros municipios de Chihuahua donde el partido hegemónico (PRI) perdió por primera vez su "preeminencia" en el poder en las elecciones de 1983, y la "ventana a la democracia (...) se cerró después del triunfo del PAN en las elecciones intermedias de 1985" (Borun- 
da, 2008, p.106). Con este precedente, podemos distinguir en la historia electoral de la ciudad vaivenes entre los partidos políticos previamente mencionados. Para cerrar la división de grandes momentos políticos de la ciudad, la tercera transición se dio a principios de 1990 con el reconocimiento de los triunfos de la oposición que se gestaron al inicio de esa década (Borunda, Paniagua y Camargo, 2017, p.29).

La legitimidad de las elecciones es otra de las preocupaciones para los estudiosos del tema electoral a nivel local. Ésta es cuestionada debido al bajo porcentaje de participación ciudadana entre 1983 y 2016: son pocas las elecciones (4 de 13) donde se ha superado el 50 por ciento de participación electoral (Borunda, Paniagua y Camargo, 2008, p.29). Para concluir, en la actualidad el gobierno de la ciudad recae en la figura de un candidato independiente que se alzó con el triunfo en el 2016 y lo ratifico en las elecciones locales de 2018, después de un largo proceso de impugnación frente al candidato del partido Morena.

\section{Metodología y bases de datos}

Las investigaciones de participación electoral son un tema discutido ampliamente y desde diversos enfoques. Desde la perspectiva geográfica se han hecho avances importantes, fundamentalmente desde el punto de vista empírico. Este trabajo hace uso de una estrategia de investigación que busca, en primer lugar, describir los patrones de asociación espacial y, en segundo lugar, revelar el efecto de "contagio espacial" provocado por el aumento o la reducción de las diferencias espaciales. Emprender una tarea como la expuesta tuvo sus retos; pero, la principal dificultad fue obtener las variables adecuadas para caracterizar adecuadamente la segregación en la ciudad. Por eso, dentro del conjunto de las bases de datos públicos disponibles se recurrió a las siguientes fuentes de información: 1) en primer lugar, los resultados de la elección de presidente de los Estados Unidos Mexicanos de 2012 (a nivel de sección electoral), obtenidos en la página electrónica del Instituto Nacional Electoral (INE, 2012), y 2) la base de estadísticas censales a escala geoelectoral del Instituto Nacional de Estadística y Geografía (INEGI, 2012).

Uno de los aportes metodológicos de este trabajo es recurrir a las secciones electorales como unidad de análisis, no se localizó un antecedente que empleara esta unidad de información. Otro apunte metodológico está relacionado con la temporalidad del más reciente Censo General de Población y Vivienda realizado en el 2010 y las elecciones federales del año 2012 que en términos generales se considera una ventaja tener datos en una ventana de tiempo casi simultánea.

El artículo combina tres métodos que incluyen: 1) análisis factorial, 2) técnicas de análisis exploratorio de datos espaciales y 3 ) un modelo de regresión geográficamente ponderado.

\subsection{Elaboración de los índices}

a) Índice compuesto de diferenciación socioespacial (ICDSE)

La metodología para la construcción del ICDSE fue tomada de Alegría (1994), Fuentes y Hernández (2013), López y Peña (2017) y De Haro (2018a). En primer 
lugar, se estimaron los componentes principales que muestran una síntesis de las variables, mediante el análisis factorial. El resultado de la técnica constituye el peso de cada variable del componente, y éste es el factor de ponderación para estimar el valor de diferenciación socio espacial de cada sección electoral. En el cuadro 1 se exponen los indicadores incluidos en la generación del valor índice, así como sus estadísticos.

Donde:

$$
I C D S E_{j}=\sum_{n=1}^{N} F P_{j} \frac{X_{i j}-\bar{X}}{\delta}
$$

$I C D S E_{j}=$ índice de diferenciación socio espacial de cada sección electoral $F P_{j}=$ Componente de la variable I, resultado del análisis factorial

$X_{i j}=$ valor de la variable $\mathrm{i}$ en la sección electoral $\mathrm{j}$

$\bar{X}=$ Media de la variable $\mathrm{i}$

$\delta=$ desviación estándar de la variable $\mathrm{i}$

Tabla 1. Estadísticas de las variables seleccionadas para la construcción del Índice de diferenciación socio espacial

\begin{tabular}{|l|c|c|c|c|}
\hline \multicolumn{1}{|c|}{ Variables } & Media & Mínimo & Máximo & Des. Est. \\
\hline Porcentaje de población de 18 años y más & 61.5 & 39.4 & 83.8 & 5.6 \\
\hline Porcentaje de población de 60 años y más & 7.7 & 0 & 25.2 & 5.1 \\
\hline Porcentaje de 15 años y más analfabeta & 1.8 & 0 & 12.4 & 1.4 \\
\hline Porcentaje de 15 años y más sin escolaridad & 3.0 & 0 & 11.9 & 2.1 \\
\hline Grado promedio de escolaridad & 9.0 & 6.0 & 14.7 & 1.7 \\
\hline Porcentaje de población nacida en la entidad & 63.4 & 31.2 & 83.7 & 7.7 \\
\hline $\begin{array}{l}\text { Porcentaje de población sin derechohabiente a } \\
\text { servicios de salud }\end{array}$ & 26.4 & 5.3 & 54.7 & 8.0 \\
\hline Porcentaje de población con religión católica & 69.4 & 40.4 & 87.6 & 6.7 \\
\hline Porcentaje de población económicamente activa & 55.7 & 37.8 & 74.0 & 5.9 \\
\hline Porcentaje de población ocupada & 52.1 & 35.7 & 71.1 & 6.1 \\
\hline Porcentaje de viviendas habitadas & 76.2 & 14.1 & 97.2 & 10.1 \\
\hline Promedio de ocupantes en viviendas habitadas & 3.4 & 1.4 & 4.9 & 0.5 \\
\hline Porcentaje de viviendas habitadas sin servicios & 8.8 & 0 & 99.8 & 9.5 \\
\hline Porcentaje de vivienda habitada sin lavadora & 40.8 & 12.9 & 92.1 & 11.1 \\
\hline Porcentaje de vivienda habitada sin automóvil & 55.0 & 22.4 & 94.9 & 12.4 \\
\hline Porcentaje de vivienda habitada sin computadora & 75.7 & 32.0 & 98.5 & 12.8 \\
\hline Porcentaje de vivienda habitada sin teléfono & 66.1 & 30.7 & 100 & 13.1 \\
\hline
\end{tabular}

Fuente: Elaboración propia con datos de INEGI (2012).

b) Índices de autocorrelación global y local

Para iniciar con la definición de los índices de autocorrelación es importante destacar que autocorrelación y dependencia espacial son conceptos que significan lo mismo: el primero es una técnica y se refiere a un fenómeno; el segundo es la explicación teórica sustentada en la primera ley de la geografía formulada por Tobler (1970, p.236) y se resume de la siguiente manera: "todo está relacionado con todo lo demás, pero las cosas cercanas están más relacionadas que las lejanas". Otros autores (Vilalta, 2006, p.91; Anselin, 1992) a partir de los aportes de Tobler propo- 
nen que el valor de una variable en una unidad es parcialmente función de la misma variable en unidades vecinas. En la literatura existen varios procedimientos estadísticos para contrastar la autocorrelación espacial; por ejemplo, la I de Moran, la C de Geary y la K de Ripley (Weichelt, 2018).

En este trabajo se hace uso del I de Moran para probar la autocorrelación a nivel global. La autocorrelación positiva significa que las áreas cercanas, en este caso las secciones electorales, tienen similares valores lo que indica una agrupación (Anselin, 1992, 1995, 1999; Krivoruchko, 2011; Chun y Griffth, 2013). En consecuencia, puede presentarse las siguientes situaciones: a) cuando los valores de las secciones electorales son similares el I de Moran será alto y positivo; b) en cambio, cuando los porcentajes de participación electoral de las secciones electorales vecinas son diferentes el I de Moran será negativo. Lo mencionado previamente, alude a dos tipologías de autocorrelación espacial, primero, la de tipo positiva o clústeres [altoalto y bajo-bajo], es decir, zonas donde los valores de los vecinos se parecen entre ellos ya sea por ser valores altos o bajos, y, en segundo término, dos variedades de autocorrelación espacial negativa [alto-bajo y bajo-alto], en otras palabras, que las secciones contiguas tiene tanto valores altos como bajos en cada uno de los indicadores evaluados (Anselin, Syabri y Smirnov, 2002; Chun y Griffith, 2013). Por consiguiente, la dispersión de los valores de la característica plasma la condición de aleatoriedad (Griffith, 1992, 1996). Esta prueba es fundamental para definir una regresión espacial (Ward y Gleditsch, 2008).

A través del gráfico de dispersión del I de Moran se puede valorar como los casos individuales contribuyen al indicador, se toma en cuenta el promedio de la variable "y" $(\bar{y})$ contra las "y es" de las unidades vecinas $\left(\bar{y}^{v}\right)$, ambas variables son estandarizadas de manera que tiene media cero y desviación estándar uno. En este gráfico de dispersión las unidades se distribuyen en cuatro cuadrantes alrededor de las medidas de $\bar{y}, \bar{y}^{v}$. Además de la distribución en cuatro cuadrantes, el gráfíco ajusta una línea de regresión donde la pendiente corresponde al valor del I de Moran. El rezago espacial (spatial lag) se define como la media de los valores de los elementos geográficos adyacentes) se ubica en el eje vertical, mientras que en el eje horizontal se colocan los valores estandarizados de cada observación (Ward y Gleditsch, 2008, pp.23-24).

El uso práctico de mayor aprobación para la autocorrelación es como instrumento de diagnóstico, puesto que tiene significativos efectos estadísticos en los modelos inferenciales porque tiende a sesgar el valor de los coeficientes del modelo de regresión clásica (Anselin, 1999; Anselin y Hudak, 1992; Anselin, Syabri, y Smirnov, 2002). El término alude a información redundante. Si "x" y " $y$ " están cabalmente correlacionadas en el espacio significa que, si se conoce el valor de " $\mathrm{x}$ " también se conocerá a "y", este grado de comprensión o correlación disminuye a medida que el I de Moran se desplaza hacia cero (Griffith, 1992; 1996). A consecuencia de las características anteriores la autocorrelación espacial es muy importante cuando se trabaja con los datos georreferenciados. Por esta razón la justificación del uso del indicador se sintetiza de la siguiente manera: el valor de una variable en un lugar definido se puede estimar a partir de los valores de las variables de los lugares vecinos (Griffith, 2009).

Para valorar la dependencia espacial se utiliza una ponderación de pesos espaciales (W), en la matriz de pesos cada dato (i) se conecta con el grupo de datos adyacentes $(\mathrm{j})$, por lo tanto, se obtiene lo que se conoce como una matriz del tipo 
Wij (Hernández, 2015). El criterio para admitir o rechazar las hipótesis de trabajo es el siguiente:

- $\mathrm{H}_{0} \mathrm{IM}=0$ el coeficiente de autocorrelación global (IM) es igual a cero, en consecuencia, no hay suficiente evidencia de un patrón espacial, y en ese sentido se esperaría un patrón aleatorio de los indicadores de participación electoral y del ICDSE.

- $\mathrm{H}_{1} \mathrm{IM} \neq 0$ el coeficiente de autocorrelación global (IM) es diferente a cero, de ahí que se afirma que existe autocorrelación espacial, entonces, es posible atestiguar que los resultados obtenidos de los indicadores de participación electoral y del ICDSE no se distribuyen aleatoriamente en el espacio.

No obstante, el I de Moran es un índice global y para representar y valorar estadísticamente arreglos locales se emplean otro tipo de estadísticos, por ese motivo para estimar los clústeres de las variables de segregación se propone utilizar los indicadores locales de asociación espacial (LISA, por sus siglas en inglés Local Indicator of Spatial Association). De manera muy general, la mecánica de los indicadores locales es desagregar geográficamente el valor del I de Moran. La prueba pretende encontrar variaciones estadísticamente significativas en el área de estudio. En el mismo sentido que el índice global estos indicadores locales pueden adquirir valores positivos y estadísticamente significativos que nos van a indicar la representación de un clúster (una sección electoral similar a sus vecinas). En cambio, un signo negativo apunta que una sección electoral tiene valores diferentes a sus vecinas, es decir, un outlier. La representación gráfica manifiesta valores atípicos espaciales cuya intensidad radicará de la significancia estadística (Getis y Ord, 1992; Anselin, 1995; Ord y Getis, 1995; Unwin, 2000).

c) Análisis de regresión

En primer lugar, se formuló un modelo de regresión simple donde la variable dependiente corresponde al porcentaje de participación electoral en cada una de las secciones electorales, y se definió mediante la siguiente formula:

$$
P_{p e}=\frac{\text { Tot Vot }_{j}}{{\text { List } \text { Nom }_{j}}_{10}} * 100
$$

Donde:

$P_{p e}=$ Porcentaje de participación electoral de cada sección electoral

Tot $V_{o t} t_{j}=$ Total de votos registrado en cada sección electoral

List $\mathrm{Nom}_{j}=$ Lista nominal de cada sección electoral

La variable independiente correspondió al resultado del análisis factorial, denominado índice comparado de diferenciación socio espacial. El modelo se estimó mediante el método de mínimos cuadrados ordinarios (MCO) y manera general:

$$
y_{i}=\beta_{0}+\beta_{1} x_{1}+\epsilon_{i}
$$


A partir de la estimación del modelo MCO y siguiendo lo sugerido por Anselin (1988) y retomado por otros autores (Ward y Gleditsch, 2008), posteriormente se realizaron las pruebas para verificar si efectivamente existe dependencia espacial sustantiva. En tercer lugar, se definieron dos modelos de regresión espacial ${ }^{4}: 1$ ) modelo de desfase o rezago espacial en la variable dependiente, conocido en inglés como spatial lag, que en una de sus primeras definiciones Anselin (1992) lo llamó proceso espacial autorregresivo (SAR), y 2) el modelo de error espacial, en inglés spatial error definidos de la siguiente manera:

$$
y_{i}=\rho \omega+\beta_{0}+\beta_{1} x_{1}+\epsilon_{i} \quad \text { Rezago espacial }
$$

Donde $\rho \omega$ expresa el coeficiente del desfase espacial que capta los efectos de desbordamiento (spillovers) de la participación electoral de las secciones electorales adyacentes, definidas mediante una matriz de contigüidad de primer orden. La regresión de rezagos espaciales es muy utilizada en ciencias sociales, debido a su carácter "endógeno" y para su estimación se emplea el método de máxima verosimilitud (Ward y Gleditsch, 2008).

$$
y_{i}=\beta_{0}+\beta_{1} x_{1}+\epsilon_{i} ; \epsilon_{i}=\lambda \omega \varepsilon \quad \text { Error espacial }
$$

Donde $\lambda \omega \varepsilon$ expresa que el término del error asociado con la matriz de contigüidad sobrelleva los efectos del modelo. La concurrencia de las variables endógenas desfasadas espacialmente requiere técnicas de estimación especiales, tales como la estimación por máxima verosimilitud y la estimación por medio de variables instrumentales (Anselin, 1988). El término de error en una regresión contiene todos los elementos ignorados desde la formulación del modelo de investigación, si estos muestran un patrón espacial significativo; por una parte, indica que nuevas variables pueden ser incluida en el modelo; por otra parte, la autocorrelación de los errores viola uno de los supuestos básicos del método de $\mathrm{MCO}$, el supuesto de errores no correlacionados. Cuando se ignora este resultado, las estimaciones realizadas con MCO serán ineficientes (Anselin, 1992).

Finalmente se estimó una regresión geográficamente ponderada para valorar la no estacionariedad del modelo de investigación. A diferencia de los modelos globales donde se asumen como estacionarios (constantes) los coeficientes, los modelos ponderados geográficamente toman en cuenta el valor de las coordenadas geográficas $\left(\mathrm{u}_{1}, \mathrm{v}_{1}\right)$ para la estimación de los coeficientes. Por lo tanto, la ecuación se define:

$$
y_{i}=\beta_{0}\left(u_{1} v_{1}\right)+\sum \beta_{1}\left(u_{1} v_{1}\right) x_{1}+\epsilon_{i}
$$

4 La definición de un modelo de regresión espacial permite ir desde un modelo global a un análisis local obteniendo un mayor grado de detalle y precisión (Lloyd y Shuttleworth, 2005). Los modelos espaciales estiman coeficientes locales de determinación para cada unidad de análisis a partir del conjunto de observaciones vecinas de esa manera se obtiene un ajuste específico para cada localización (Fotheringhm Brunsdon y Charlton, 2002). La desagregación del coeficiente de determinación global en " $n$ " coeficientes locales, aunado al análisis de su distribución geográfica permitirá identificar dónde la(s) variable(s) independiente(s) tienen mayor o menor explicación (Fotheringhm et al., 2002; Lloyd y Shuttleworth, 2005). 
Para calcular la influencia de las secciones electorales vecinas se utiliza una matriz de pesos, por consiguiente, la evaluación de los coeficientes depende de las observaciones de la función de ponderación y de lo que se conoce como el ancho de banda. En la estimación de este modelo se utilizó un ancho de banda adaptativo que facilita el cálculo y la búsqueda de un número específico de secciones electorales vecinas para garantizar un valor de muestra locales óptimas. El modelo puede lograr un mayor rendimiento a diferencia de los modelos de regresión tradicionales y la lectura de los coeficientes puede guiar a nuevas interpretaciones de los fenómenos (Nakaya, 2016).

\section{Resultados y análisis}

El análisis empírico de la relación entre el ICDSE y la participación ciudadana (Figura 1) proporciona valiosos factores para valorar el peso específico que tienen las condiciones socioeconómicas y de diferenciación espacial en la participación de las elecciones del año 2012.

Figura 1. Porcentajes de participación electoral, índice compuesto de diferenciación socio espacial, Ciudad Juárez 2010-2012

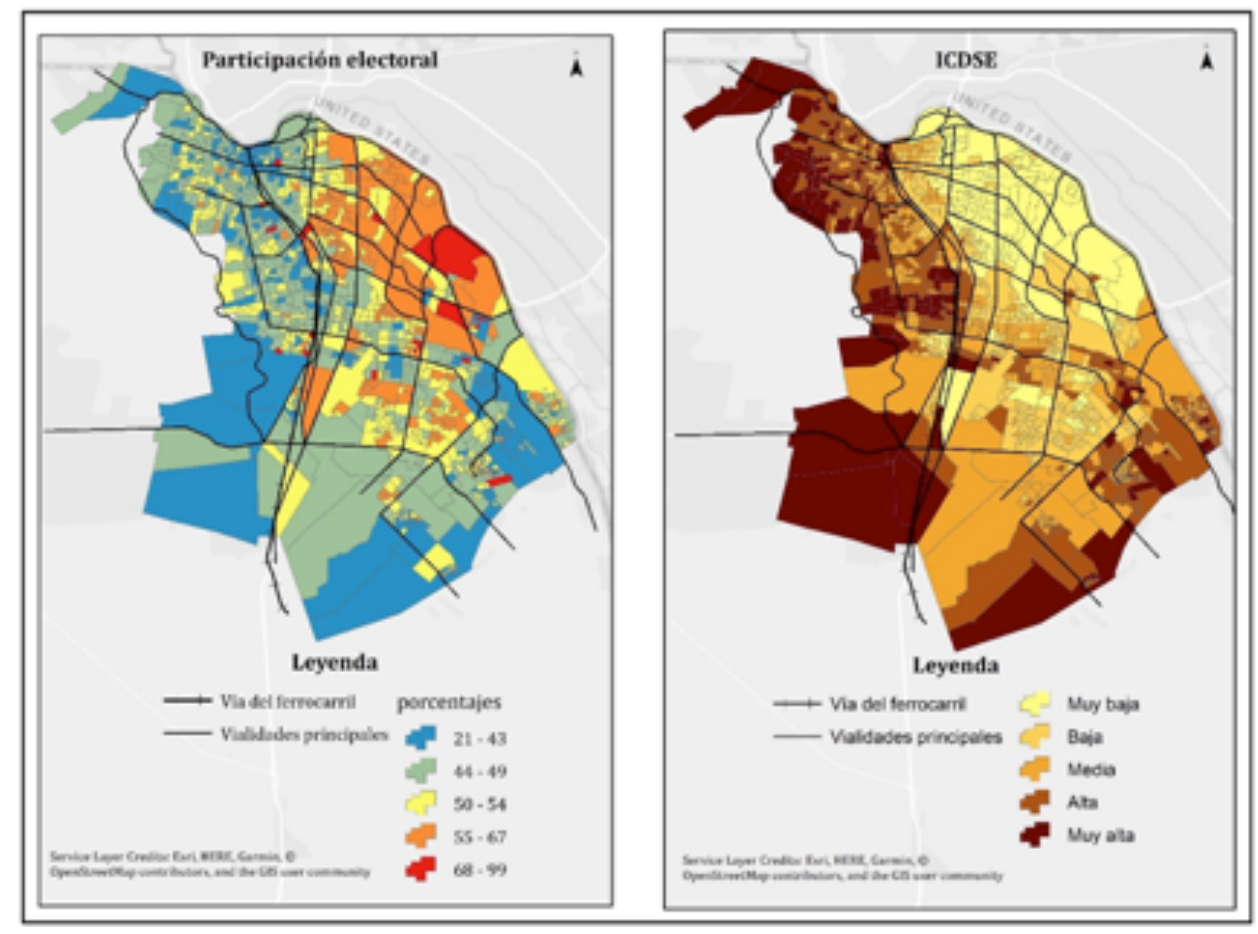

Fuente: Elaboración propia con datos de INEGI (2012); INE (2012). 
El nivel de participación electoral en las 1,107 secciones electorales analizadas registro una media de 48.72 por ciento, una desviación estándar de 7 , la sección con el registro mínimo alcanzó el 21.36 y la máxima de 98.84 por ciento.

El resultado de nivel de participación se agrupo en cinco categorías. Existe una franja que se ubica de norte a sur comprendida entre la carreta federal 45 y la vía del tren que funciona como un espacio de transición entre el poniente y oriente de la ciudad, en trabajos previos (Fuentes y Hernández, 2013, p.56) se evidencia que las mejores condiciones de accesibilidad y jerarquía socioespacial son resultado de las nuevas zonas comerciales y residenciales que se desplazaron desde el centro de la ciudad hacia el nororiente; la zona central presenta un proceso de deterioro, y rumbo al poniente de la ciudad se localizan los sectores con los menores valores de accesibilidad y jerarquía. En este sentido, los porcentajes de la participación electoral son coincidentes con la distribución espacial previamente mencionada.

En general el patrón espacial del ICDSE es similar al patrón geográfico de participación electoral, aunque el primero está un poco más desagregado; el segundo muestra patrones mucho más definidos, de igual forma los resultados se agruparon en cinco categorías que permite observar el cambio de los valores desde los más bajos - mejores condiciones - que se distribuyen desde el norponiente hasta los más bajos - peores condiciones - en la periferia de la ciudad. Uno de los principales resultados es una correlación negativa de 0.52 con la fragmentación socioeconómica de la ciudad, es decir, entre menor es la diferenciación socio espacial mayor es la participación electoral.

\subsection{Indicadores de autocorrelación espacial}

En primer lugar, los resultados de las pruebas tanto en el modelo global del I de Moran y local establecen evidencia de agrupamientos espaciales, lo que significa que el espacio geográfico los valores de porcentajes y de diferenciación socio espacial tiene una mayor relación con aquellos espacios más cercanos que los distantes. En cuanto a la prueba global en el porcentaje de participación electoral se obtuvieron los siguientes valores el I de Moran es de 0.2574, mientras que en el caso del ICDSE el valor se incrementa hasta 0.6841 , en ambos casos se diferencian del valor esperado si la distribución hubiese sido aleatoria E[I]: -0.0009 con un pseudo pvalue de -0.001 , esto es, que la probabilidad de que el resultado sea consecuencia del azar es muy baja. La información de los índices locales se presenta por cuadrantes para facilitar su análisis (Figura 2).

La primera observación de los indicadores locales de autocorrelación espacial reafirma la fragmentación de las variables en el espacio de la ciudad. En cuanto al análisis de la participación ciudadana el cuadrante 2 (Alto - Alto) concentra el 16.8 por ciento de las secciones electorales, aunque este valor es ligeramente inferior al porcentaje del cuadrante 3 (Bajo - Bajo con $18.3 \%$ de las secciones) se agrupa principalmente en la zona norponiente de la ciudad; mientras que los valores bajosbajos de participación electoral muestran una distribución dispersa por la periferia de la ciudad. Los cuadrantes 1 y 4 con valores bajo-alto y alto, bajo, tienen el 3.9 y 3.8 por ciento de las secciones electorales respectivamente. Por lo que respecta al ICDSE la separación entre las unidades de análisis se hace todavía más evidente, de manera que existe una alta segregación en la zona de estudio, puesto que la mayoría de las secciones del cuadrante 1 (Alto - Alto 38.7 \%) conforman un cinturón 
desde el poniente hasta el suroriente de la ciudad. En contraste, el cuadrante 3 (Bajo - Bajo) 27.7 por ciento de las secciones se agrupan en dos zonas de la ciudad: norponiente y sur.

Figura 2. Indicadores locales de autocorrelación espacial de la participación electoral, índice compuesto de diferenciación socio espacial, Ciudad Juárez 2010-2012

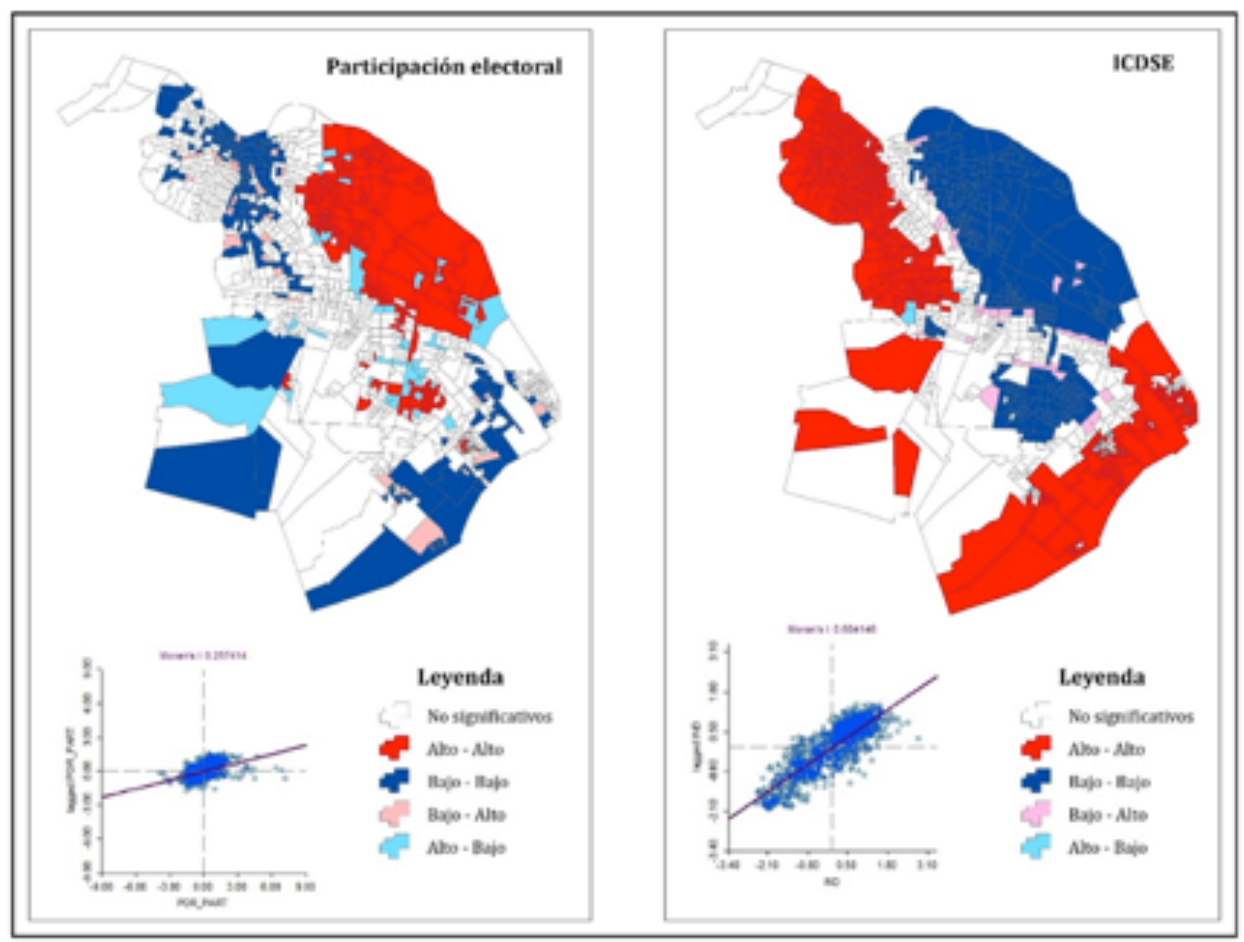

Fuente: Elaboración propia con datos de INEGI (2012); INE (2012).

Del mismo modo se realizó una estimación de autocorrelación espacial bivaria$\mathrm{da}$, en el cual el ICDSE se colocó en el eje de las " $\mathrm{x}$ " y el porcentaje de participación electoral en el de las " $y$ ". El resultado del I de Moran es de 0.3545 , diferente del valor esperado si la distribución hubiese sido aleatoria $\mathrm{E}[\mathrm{I}]:-0.0009$ con un pseudo p-value de -0.001, es decir, la probabilidad de que el resultado sea producto del azar es muy baja.

Klos (2008) hizo uso del indicador bivariado de autocorrelación espacial para verificar el cambio del comportamiento del voto y el valor medio de las viviendas en dos condados de Pensilvania (Estados Unidos), y encontró que a medida que el valor aumenta existe un ligero agrupamiento de comportamiento a favor del voto demócrata. Como menciona Anselin (1992) la comprobación de un agrupamiento espacial es sólo un primer paso del análisis de los datos espaciales, en ese sentido, la comprobación de la autocorrelación es muy imprecisa para ser útil en la construcción teórica. 


\subsection{Análisis de regresión}

Lo analizado hasta el momento ha demostrado los valores de dependencia espacial de los dos indicadores del trabajo. A partir de la estimación del modelo mediante MCO y siguiendo lo sugerido por Anselin (1988), primero se ha estimado un modelo de regresión por el método de mínimos cuadrados ordinarios, y posteriormente se han realizado las pruebas para verificar si efectivamente existe dependencia espacial sustantiva (Tablas 1 y 2). A continuación, se discuten los resultados del análisis de regresión del modelo estimado mediante la técnica de mínimos cuadrados ordinarios.

Tabla 2. Modelo de regresión simple relativo a la participación electoral, Ciudad Juárez 2010-2012

\begin{tabular}{|l|c|c|c|c|}
\hline \multicolumn{5}{|c|}{ Variable dependiente porcentaje de participación electoral } \\
\hline \multicolumn{1}{|c|}{ Variables } & Coeficientes & Error estándar & Estadístico $t$ & Significancia \\
\hline Intercepto & 48.7198 & 0.1788 & 272.395 & 0.000 \\
\hline ICDSE & -0.860175 & 0.0419 & -20.4961 & 0.000 \\
\hline \multicolumn{3}{|l|}{} & Estadístico: & Probabilidad: \\
Número de observaciones: 1,113 & & Jarque- Bera & 0.0000 \\
Número de variables: 2 & & Breusch-Pagan test & 0.6762 \\
R2: 0.2743 & & Koenker-Bassett & 0.8916 \\
R2 ajustada: 0.2737 & & I Moran residuales & 0.0000 \\
\hline
\end{tabular}

Fuente: Elaboración propia con datos de INEGI (2012); INE (2012).

El modelo explica el 27.4 por ciento de la variabilidad de la participación electoral. Dado que se trata de un modelo simple no se presentan problemas de multicolinealidad, la variable explicativa o independiente es estadísticamente significativa; sin embargo, el valor de las pruebas de heterocedasticidad (Breusch-Pagan 0.6776 y Koenker-Bassett 0.8916 mayores a 0.05 ) indican que el modelo presenta no estacionariedad estadísticamente significativa, es decir, el modelo no es consistente en el espacio geográfico. En consecuencia, la diferenciación socioespacial no se comporta de la misma manera en la zona de estudio. En cuanto a la prueba de los residuales, el resultado del estadístico Jarque-Bera indica una probabilidad de 0.0 menor a 0.05 , lo que significa que los residuales no son distribuidos normalmente, lo anterior se complementa con el cálculo del I de Moran a los residuales obteniendo un valor de 0.1059 con un pseudo p-value de -0.001 , esto es, que la probabilidad de que el resultado (clúster) sea consecuencia del azar es muy baja.

Los resultados parciales indican que la regresión por $\mathrm{MCO}$ no cumple con la condición de distribución idéntica e independiente de los errores; en consecuencia, se estimaron dos modelos de regresión espacial y un modelo ponderado geográficamente para valorar la no estacionariedad del fenómeno. El modelo de regresión de rezago espacial (spatially lagged) es apropiado cuando existe evidencia que los valores de "y" de una unidad están directamente influyendo en los valores "y" de sus vecinos (Ward y Gleditsch, 2008, p.35). 
Tabla 3. Modelos de regresión simple relativo a la participación electoral, Ciudad Juárez 2010-2012

\begin{tabular}{|l|c|c|c|}
\hline & MCO & Rezago & Error \\
\hline Constante & 48.7198 & 33.02 & 48.8473 \\
\hline ICDSE & -0.860175 & -0.672074 & -0.875743 \\
\hline $\mathrm{R}^{2}$ & 0.274373 & 0.303032 & 0.318894 \\
\hline $\mathrm{R}^{2}$ ajustada & 0.273720 & & \\
\hline$\rho$ & ------ & 0.323118 & 0.441085 \\
\hline$\lambda$ & ------ & ----- & \\
\hline
\end{tabular}

Fuente: Elaboración propia con datos de INEGI (2012); INE (2012).

Los resultados del modelo de rezago espacial confirman lo analizado en la sección de autocorrelación espacial, el ICDSE tiene el signo esperado (negativo) confirmado la relación directa e inversa evidenciada previamente. En ese sentido la explicación es la siguiente: si se da una disminución de una unidad en el nivel de diferenciación socioespacial entonces aumenta el nivel de participación electoral en 0.67 puntos. Es muy importante señalar que estos valores varían en el espacio urbano por la incidencia de la matriz de multiplicadores espaciales en la proporción del coeficiente $\rho$, que en el modelo es de 0.3231 estos resultados nos permiten rechazar la hipótesis nula, verificando que la configuración espacial de la concentración espacial de la participación ciudadana en Ciudad Juárez durante las elecciones presidenciales del 2012 no se produce de manera aleatoria quedando evidencia de la interdependencia y retroalimentación entre las secciones electorales ratificando el efecto de "vecindario".

Los resultados del análisis del modelo de regresión geográficamente ponderado manifiestan la relación espacial de los coeficientes valorados localmente. En este modelo la condición de no estacionariedad se revela a través de la comparación entre las estimaciones de los rangos locales y el intervalo de confianza alrededor de la estimación global. De acuerdo con la literatura revisada cuando el rango estimado del modelo local es mayor a más, menos una desviación estándar del parámetro estimado global se establece que la relación espacial es de tipo no estacionaria (Fotheringham et al., 2002). En las tablas 4 y 5 se muestran los resultados de los modelos global y local.

Tabla 4. Síntesis del modelo de regresión global

\begin{tabular}{|c|c|c|c|}
\hline Variable & Coeficiente & Error estándar & $t$ \\
\hline Intercepto & 48.750914 & 0.179019 & 272.323083 \\
\hline ICDS & -0.868676 & 0.042059 & -20.653931 \\
\hline
\end{tabular}

Fuente: Elaboración propia con datos de INEGI (2012); INE (2012).

En la Tabla 5 el rango del ICDSE fue de 1.303145, este es más grande que el valor de la desviación estándar del parámetro estimado global de 0.042059 , lo que 
ratifica que la diferenciación participación electoral en Ciudad Juárez en las elecciones de 2012, es espacialmente no estacionario.

Tabla 5. Síntesis del resultado de coeficientes del modelo RGPG

\begin{tabular}{|c|c|c|c|c|c|}
\hline Variable & $\begin{array}{c}\text { Coeficientes } \\
\text { mínimos }\end{array}$ & $\begin{array}{c}\text { Cuartil más } \\
\text { bajo }\end{array}$ & Rango & $\begin{array}{c}\text { Cuartil más } \\
\text { alto }\end{array}$ & $\begin{array}{c}\text { Coeficientes } \\
\text { máximos }\end{array}$ \\
\hline Intercepto & 44.9282204 & 47.218680 & 6.319780 & 48.804886 & 51.247983 \\
\hline ICDSE & -1.438459 & -1.013593 & 1.303145 & -0.523940 & -0.135314 \\
\hline
\end{tabular}

Fuente: Elaboración propia con datos de INEGI (2012); INE (2012).

En la Tabla 6 se presentan dos medidas de banda de ajuste para ambos modelos gaussianos. En primer lugar, el valor del coeficiente de correlación local muestra que este mejoró en 7 por ciento las estimaciones del modelo global. Otro indicador importante es el criterio de información Akaike (AICc), este criterio se compara entre los modelos estimados y el modelo con el menor valor expresa en mejores términos la variación del ICDS con respecto a la participación electoral, en este caso corresponde al modelo local con un valor de 7008.25 menor a 7096.22 del modelo global.

Tabla 6. Medidas de banda de ajuste del modelo global y local

\begin{tabular}{|l|c|c|}
\hline \multicolumn{1}{|c|}{ Modelo } & Coeficiente de correlación & AICc \\
\hline Gaussiano global & 0.27 & 7096.229865 \\
\hline Gaussiano local & 0.34 & 7008.258712 \\
\hline
\end{tabular}

Fuente: Elaboración propia con datos de INEGI (2012); INE (2012).

Los coeficientes locales, como quedó debidamente explicado, manifiestan una no estacionariedad espacial, en la Figura 3 se muestran como estos valores se distribuyen a lo largo y ancho de las secciones electorales. Los resultados se agruparon en cinco categorías que permite observar el cambio de los coeficientes locales desde 1 hasta 49 por ciento. El patrón geográfico de los coeficientes es más alto (40 al $49 \%$ ) en la zona nororiente de la ciudad y disminuye hacia las periferias. En la zona norponiente de la ciudad donde el índice comparado de diferenciación socio espacial es más bajo explica hasta en 49 por ciento la participación electoral y tiene menos valor explicativo en la zona oriente y suroriente de la ciudad.

Por consecuente, el espacio manifiesta una relación entre los procesos y funciones que determina la participación electoral con las condiciones desiguales que se presentan. Demostrando como el contexto de ciudad fronteriza evidencia esos cambios constantes en su composición socio espacial y político electoral. De acuerdo con De Haro (2018a), Ciudad Juárez manifiesta condiciones de carácter urbano, social, económico y político que han estado presentes en sus procesos. Entre estos procesos se evidencian las condiciones del rezago social consecuencia de un crecimiento poco planeado territorialmente, situándose la población en su mayoría migrante en espacios lejanos de la ciudad y sin movilidad urbana. Las viviendas de interés social en la periferia eran de bajo costo para los trabajadores 
de la industria maquiladora, por lo que, su crecimiento fue creando espacios que muestran desigualdad e inseguridad. Por lo que, el interés de la participación político electoral se ha acompañado por este tipo de población vulnerable.

Figura 3. Coeficientes de determinación locales, regresión geográficamente ponderada, participación electoral y diferenciación socio espacial, Ciudad Juárez 2010-2012

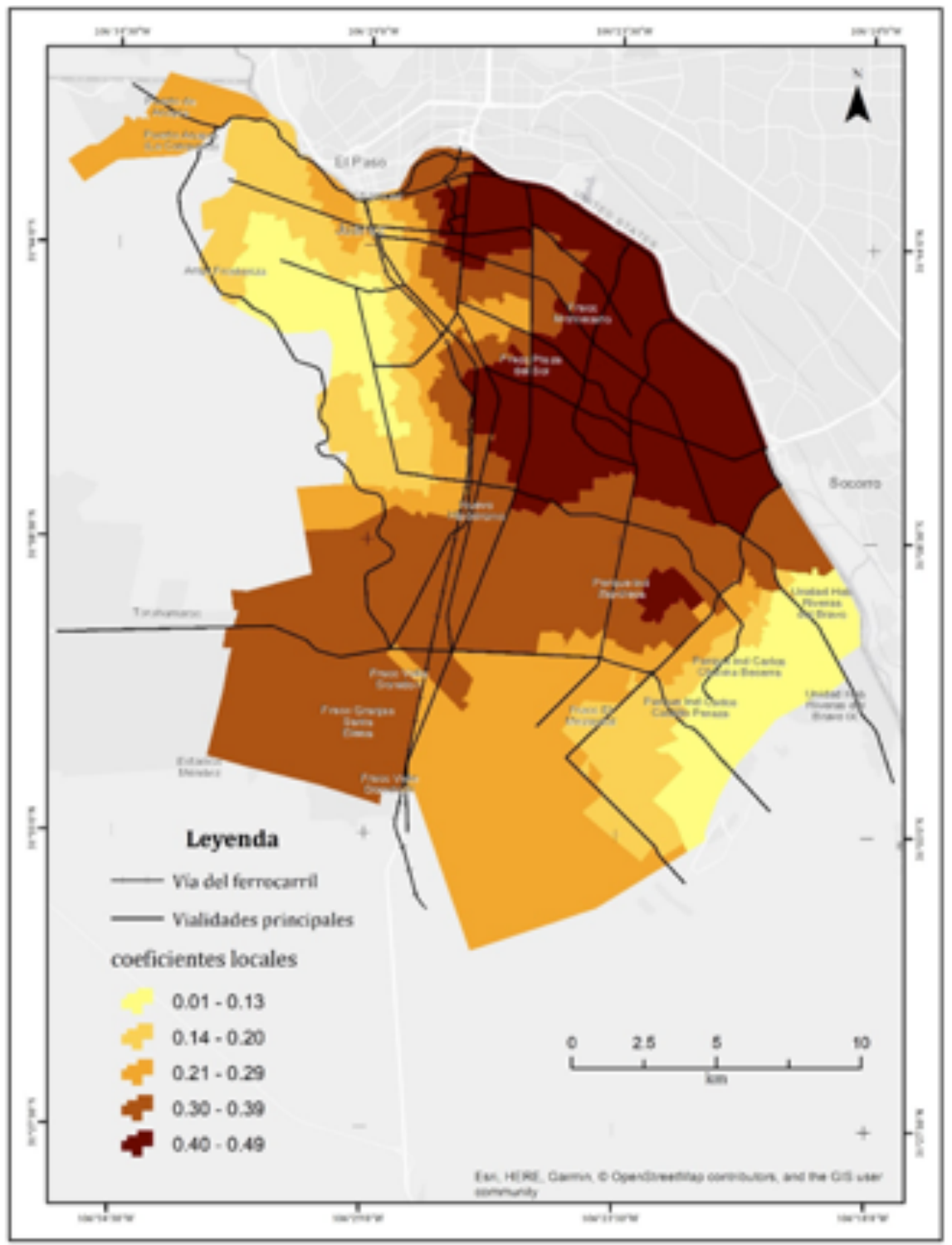

Fuente: Elaboración propia con datos de INEGI (2012); INE (2012).

Aunque la participación electoral no es del todo representativa entre los tres niveles de gobierno como muestran los resultados, existe una historia que impulsó 
dicha participación ciudadana en el régimen democrático. Es por ello, que la conducta del electorado dependerá de sus condiciones socioespaciales. Por otro lado, estas condiciones impactan en la cuestión sociopolítica, como se menciona en el contexto, Juárez representa en su mayoría población migrante donde el arraigo o sentido de pertenencia al lugar no interesa. Asimismo, el nivel de inseguridad y violencia que presenta se refleja en el poco interés que la ciudadanía tiene por participar en asuntos de índole política.

\section{Conclusiones}

En primer lugar, este trabajo es pionero, al menos en el contexto nacional, en estimar un modelo espacial mediante el uso de las unidades de menor tamaño a escala geo-electoral. En el mismo sentido, se realizó la estimación de un indicador de diferenciación socioespacial y de esta manera se contó con información estadística en la misma escala de análisis lo cual representó un importante avance metodológico. También es muy importante mencionar que existe un importante reto en cuanto a los datos disponibles para capturar de mejor manera el índice de diferenciación socioespacial; por ejemplo, no se cuentan con la variable ingreso, clave para este tipo de índices. En cuanto a los métodos espaciales la disponibilidad de información georreferenciada ayuda a superar las limitaciones de las técnicas no espaciales para el análisis de los datos.

El trabajo evidencia una marcada polarización de la participación electoral y de la segregación socioespacial. Los principales resultados apuntan que ambos fenómenos tienen un elevado nivel de dependencia espacial. Esto permite expresar que el proceso electoral del 2012 a nivel de las secciones electorales no apunta a un proceso espacial aleatorio, sino por el contrario existen pruebas de un efecto vecindario. En este sentido los valores de las secciones electorales vecinas es una variable explicativa.

La identificación de los patrones espaciales tiene una importante lectura a partir de las condiciones de la estructura urbana de la ciudad; la segregación urbana en Ciudad Juárez parece tener un efecto en los niveles de participación electoral. Las zonas con mejores condiciones urbanas son más participativas, lo cual nos permite plantear una nueva hipótesis relacionada con los efectos del desarrollo-bienestar que podría inducir a una participación más activa en la política; por el contrario, las condiciones de rezago social inhiben la participación debido a la necesidad primero de satisfacer necesidades básicas y en segundo lugar o en menor medida pensar en la participación electoral. En este sentido, retomanos la mención que hace PérezCampuzano (2011, p.407) de que la segregación es un tema de acceso y poder en distintas esferas de la vida,. En este caso la participación electoral, y su manifestación espacial, es precisamente la expresión de las oportunidades y de poder con el que los grupos cuenten; en consecuencia, la organización espacial es el resultado de la capacidad de decidir y del poder para llevarlas a cabo.

Otro de los aportes del trabajo es el énfasis en la escala de análisis donde planteamos que no necesariamente los procesos se transfieren de lo nacional a lo local o viceversa, como planteaba Agnew (2007) los procesos electorales pueden enlazar a los lugares, pero también pueden separarlos en su singularidad, y, en este sentido, los resultados de este trabajo apuntan a una singularidad local debido a la baja par- 
ticipación de las zonas con altas diferencias socioespaciales. Los resultados obtenidos pueden ser importantes insumos para abordar el tema desde los enfoques normativos y de la elección racional en temas de clientelismo político, así como apoyar las hipótesis planteadas sobre la relación entre factores contextuales como la marginación y el nivel de urbanización en la decisión del votante sobre intercambiar o no su apoyo político (Tapia y Gatica, 2016, p.505). En otras palabras, los enfoques de estudio de los procesos electorales pueden complementarse para avanzar en el conocimiento. Agnew (1996) planteó que la separación entre el espacio y la sociedad no podría sostenerse, y para resolver ese dilema propone la perspectiva "del lugar" como marco teórico adecuado para acercar - cerrar la brecha- el análisis abstracto — desde la mirada sociológica — con la mirada concreta del análisis geográfico.

Para los estudios electorales en México, en particular los concernientes a la geografía electoral, en este trabajo se han aportado evidencias empíricas en un contexto complejo como es Ciudad Juárez, enclavada en una situación geográfica estratégica y con un modelo de desarrollo dependiente de su interacción con los Estados Unidos de América.

\section{Referencias}

Agnew, J. (1990). From Political Methodology to Geographical Social Theory: A Critical Review of Electoral Geography 1960-1987. En R.J. Johnston, F.M. Shelley y P.J. Taylor (Eds.), Developments in Electoral Geography (pp.15-21). Londres: Routledge.

Agnew, J. (1996). Mapping politics: how context counts in electoral geography. Political Geography, 15(2), 129-146.

Agnew, J. (2002). Place and Politics in Modern Italy. Chicago, IL: University of Chicago Press.

Agnew, J. (2007). Remaking Italy? Place configurations and Italian Electoral Politics Under the Second Republic, Modern Italy, 12(1), 17-38.

Aldrich, J. (1993). Rational Choice and Turnout. American Journal of Political Science, 37(1), 246-278.

Alegría, T. (1994). Segregación socioespacial urbana. El ejemplo de Tijuana. Estudios Demográficos y Urbanos, 9(2), 411-428.

Anselin, L. (1988). Spatial Econometrics: methods and models. Boston: Kluwer Academic.

Anselin, L. (1992). Spatial data with GIS: An introduction to applications in the social science. Technical Report 92-10, August 1992, National Center for Geographic Informational and Analysis, University of California.

Anselin, L. (1995). Local Indicators of Spatial Association - LISA. Geographical Analysis, 27(2), 93-115.

Anselin, L. (1999). Interactive Techniques and Exploratory Spatial Data Analysis. En P. Longley, M. Goodchild, D. Maguire y D. Rhind, Geographical Information Systems: Principles, Techniques, Management and Applications (pp.251-264). Nueva York: Wiley.

Anselin, L., y Hudak, S. (1992). Spatial econometrics in practice. A review of software options. Regional Science and Urban Economics, 22(3), 509-536. 
Anselin, L., Syabri, I., y Smirnov, O. (2002). Visualizing multivariate spatial correlation with dynamically linked windows. Working Paper Regional Economics Applications Laboratory, REAL 02-T-8.

Ávila-Eggleton, M., y Gutiérrez Sánchez, H. (2017). El papel del espacio en la explicación del abstencionismo. Espiral, 24(69), 115-154.

Beaugutte, L., y Colange, C. (2013). Analyser les comportements électoraux à l'échelle du bureau de vote. Francia. Recuperado de https://halshs.archives-ouvertes.fr/halshs00839899

Borunda, E. (2008). Ciudadanía, modernización y derechos políticos en Ciudad Juárez: estudio comparado de los períodos 1983 - 1986 y 2004- 2007. (Tesis de Doctorado en Ciencias Sociales con Especialidad en Estudios Regionales), El Colegio de la Frontera Norte, Tijuana, México.

Borunda, E., Paniagua, A., y Camargo, I. (2017). Las candidaturas independientes: los nuevos actores de la democracia mexicana. En E. Borunda, A. Paniagua y I. Camargo (Coords.) Procesos electorales en México. Una visión de las campañas electorales, la comunicación política, los candidatos y sus electores (pp. 15-52). México: El Colegio de Chihuahua.

Box-Steffensmeier, J., Brady, H., y Collier, D. (2008). Political Science Methodology. En J. Box-Steffensmeier, H. Brady y D. Collier (Eds.), The Oxford Handbook of Political Methodology. Nueva York, NY: Oxford University Press. DOI: 10.1093/oxfordhb/9780199286546.003.0001

Cervera, L. E., y Monárrez, J. (2013). Introducción. En L. E. Cervera y J. E. Monárrez (Coords.), Geografía de la violencia en Ciudad Juárez (pp.23-34). Chihuahua, México: El Colegio de la Frontera Norte.

Charles-Leija, H., Torres, A. J., y Colima, L. M. (2018). Características sociodemográficas del voto para diputados, 2015. Revista del Colegio de San Luis, VIII(17), 107-135.

Chen, J., y Rodden, J. (2009). Tobler's Law, Urbanization, and Electoral Bias: Why Compact, Contiguous District are Bad for the Democrats. Manuscrito no publicado. Recuperado de http://www.stanford.edu/ jrodden/florida.pdf

Chun, Y. y Griffith, D. (2013). Spatial Statistics \& Geostatistics. Londres: SAGE.

Crespin, M., Darmofal, D., y Eaves, C. (2011). The Political Geography of Congressional Elections. Annual Meeting of the Midwest Political Science Association, Chicago, IL, USA. Recuperado de http://crespin.myweb.uga.edu/CrespinDarmofalEaves.pdf

Cutts, D., y Webber, D. J. (2010). Voting patterns, Party Spending and Relative Location in England y Wales. Regional Studies, 44(6), 735-760.

Darmofal, D. (2006). Spatial Econometrics and Political Science. Annual Metting of the Southern Political Science Association, Atlanta, GA, USA. Recuperado de https://people.cas.sc.edu/darmofal/Spatial\%20Econometrics.pdf

De Haro, L. (2018a). Análisis geoelectoral. Diferenciación socioespacial en la participación electoral en Ciudad Juárez, Chihuahua (2012). (Tesis de Doctorado en Geografía y Ordenación Territorial), Universidad de Guadalajara, Jalisco, México.

De Haro, L. (2018b). Configuración geoespacial del abstencionismo electoral en el Estado de Chihuahua. Geografia y Sistemas de Información Geográfica, 10(10), 52-67.

Duncan, O. D., y Duncan, B. (1955). A Methodological Analysis of Segregation Indexes. American Sociological Review, 20(2), 210-217.

Emmerich, G. (1993). Introducción a los estudios de geografía electoral en México. Votos y mapas. México: Universidad Autónoma del Estado de México. 
Fotheringham, A. S., Brunsdon, C. y Charlton, M.E. (2002). Geographically weighted regression: the analysis of spatially varying relationships. Chichester, RU: Wiley.

Fuentes, C. (2009). La estructura espacial urbana y accesibilidad diferenciada a centros de empleo en Ciudad Juárez, Chihuahua. Región y Sociedad, 23(1), 35-56.

Fuentes, C., y Hernández, V. (2013). Segregación socioespacial y accesibilidad al empleo en Ciudad Juárez, Chihuahua (2000-2004). Región y Sociedad, 25(56), 43-74.

Fuentes, C., y Hernández, V. (2014). Housing finance reform in Mexico: the impact of housing vacancy on property crime. International Journal of Housing Policy, 14(4), 368-388, DOI: 10.1080/14616718.2014.955332

Fuentes, C., Peña, S., y Hernández, V. (2018). La medición multidimensional de la pobreza a nivel intraurbano en Ciudad Juárez, Chihuahua (2012). Estudios Fronterizos, 19. DOI: $10.21670 /$ ref. 180001

Getis, A., y Ord, J. K. (1992). The analysis of spatial association by use of distance statistics. Geographical Analysis, 24(3),189-206. DOI: 10.1111/j.1538-4632.1992.tb00261.x

Gómez-Tagle, S. (2009). ¿Cuántos votos necesita la democracia? La participación electoral en México, 1961-2006. México: Instituto Federal Electoral.

Griffith, D. A. (1992). What is spatial autocorrelation? Reflections on the past 25 years of spatial statistics. L' Espace Géographique, 21(3), 265-280.

Griffith, D. A. (1996). Spatial autocorrelation and eigenfunctions of the geographic weight matrix accompanying geo-reference data. The Canadian Geographer / Le Géographe canadien, 40(4), 351-367.

Griffith, D. A. (2009). Modeling spatial autocorrelation in spatial interaction data: empirical evidence from 2002 Germany journey-to-work flows. Journal of Geographical Systems, $11(2), 117-140$.

Guerrero, B. (2001). Geografía electoral en México. Boletín del Centro de Capacitación Judicial Electoral, 7(5), 1-7.

Harder, J., y Krosnick, J. (2008). Why Do People Vote? A Psychological Analysis of the Causes of Voter Turnout. Journal of Social Issues, 64(3), 525-549.

Hedström, P. (2008). Studying Mechanisms to Strengthen Causal Inferences in Quantitative Research. En J. Box-Steffensmeier, H. Brady y D. Collier (Eds.), The Oxford Handbook of Political Methodology. Nueva York, NY: Oxford University Press. DOI: 10.1093/oxfordhb/9780199286546.003.0013

Hernández, V. (2015). Análisis geoespacial de las elecciones presidenciales en México, 2012. EURE, 41(122), 185-207.

Hernández, V., y De Haro, L. (2017). Análisis geoespacial de la elección para gobernador en Chihuahua, México, 2010. En E. Borunda, A. Paniagua y I. Camargo (Eds.), Procesos electorales en México. Una visión de las campañas electorales, la comunicación politica, los candidatos y sus lectores (pp.53-85). México: El Colegio de Chihuahua.

Ichuta Nina, C. E. (2017). Los estudios del comportamiento electoral en el estado de Hidalgo. El rezago de una disciplina. Revista Mexicana de Estudios Electorales, 2(19), 83-120.

INE (Instituto Nacional Electoral). (2012). Sistema de Consulta de la Estadística de las Elecciones Federales 2011-2012. Recuperado de http://siceef.ife.org.mx/pef2012/SICEEF2012.html\# 
INEGI (Instituto Nacional de Estadística y Geografía). (2012). Estadísticas censales a escalas geoelectorales, Censo de Población y Vivienda 2012. Recuperado de http:/gaia.inegi.org.mx/geoelectoral/viewer.html

INEGI (Instituto Nacional de Estadística y Geografía). (2019). Estados Unidos Mexicanos. $6^{\circ}$ Censo de Población 1940. Chihuahua. Recuperado de https://www.inegi.org.mx/app/biblioteca/ficha.html?upc $=702825411824$

Jargowsky, P. A., y Kim, J. (2005). A Measure of Spatial Segregation: The Generalized Neighborhood Sorting Index. Richardson, TX: National Poverty Center. Recuperado de http://www.npc.umich.edu/publications/workingpaper05/paper03/jargowsky_kim_21ma r2005.pdf

Johnston, R. (1979). Political, Electoral and Spatial Systems. Oxford: The Clarendon Press.

Johnston, R., y Pattie, C. (2003). Representative Democracy and Electoral Geography. En J. Agnew, K. Mitchell y G. Toal (Eds.), A Companion to Political Geography (pp.335355). Oxford: Blackwell.

Johnston, R., y Pattie, C. (2006). Putting Voters in their Place: Geography and Elections in Great Britain. Oxford: Oxford University Press.

Johnston, R., Gregory, D., y Smith, D. M. (1987). Diccionario de geografia humana (2a ed.). Madrid: Alianza Editorial.

Klos, J. (2008). Determining spatial correlations between voting behavior and select demographic variables in a changing electorate. Middle States Geographer, 41, 19-26.

Krivoruchko, K. (2011). Spatial Statistical Data Analysis for GIS Users. Redlands, CA: ESRI Press.

Lefebvre, B., y Robin, C. (2009). Pre-electoral Coalitions, Party System and Electoral Geography: A Decade of General Elections in India (1999-2009). South Asia Multidisciplinary Academic Journal, 3. DOI: 10.4000/samaj.2795

Lewis-Beck, M., Jacoby, W., Norpoth, H., y Weisberg, H. (2008). The American Voter Revisited. Ann Arbor, MI: University of Michigan Press.

Lizama, G. (2012). Geografía electoral del abstencionismo en los Municipios de México (1994-2009). Espacialidades, 2(2), 22-51.

Lloyd, C. D., y Shuttleworth, I. (2005). Analysing commuting using local regression techniques: scale, sensitivity, and geographical patterning. Environment and Planning A, 37(1), 81-103.

López, J., y Peña, S. (2017). La segregación socioespacial en Ciudad Juárez, Chihuahua, 1990-2010. Región y Sociedad, 29(68), 115-152.

Madoré, F. (2004). Ségrégation sociale et hábitat. Rennes: Presses Universitaries de Rennes

Maggini, N. (2017). The Explanatory Model: The Determinants of Youth Voting Choices. Londres: Palgrave Macmillan.

Massey, D. S. (2012). Reflections on the Dimensions of Segregation. Social Forces, 91(1), 39-43.

Massey, D. S., y Denton, N. A. (1988). The Dimensions of Residential Segregation. Social Forces, 67(2), 281-315.

Mckelvey, R., y Ordeshook, P. (1986). Information, Electoral Equilibria and the Democratic Ideal. Journal of Politics, 48(4): 909-937.

Mohd, M., Azlina, A., y Mohd, I. (2016). Voting Behaviour in Malaysia: Locating the Sociological Determinants of Ethnicity, Middle Classes and Development Gains. World Applied Sciences Journal, 34(6), 805-812. 
Molinar, J. (1991). Counting the Number of Parties: An Alternative Index. American Political Science Review, 85(4), 1383-1391.

Monzón, N. B. (2009). Geografía electoral. Consideraciones teóricas para el caso argentino. Cuadernos de Geografia. Revista Colombiana de Geografia, (18), 119-128.

Morton, R., y Williams, K. (2008). Experimentation in Political Science. En J. BoxSteffensmeier, H. Brady y D. Collier (Eds.), The Oxford Handbook of Political Methodology. Nueva York, NY: Oxford University Press. DOI: 10.1093/oxfordhb/9780199286546.003.0014

Nakaya, T. (2016). GWR4. Windows Application for Geographically Weighted Regression Modelling. Recuperado de https://github.com/gwrtools/GWR4/blob/master/GWR4manual_409.pdf

O’Loughlin, J. (2002). The Electoral Geography of Weimar Germany: Exploratory Spatial Data Analyses (ESDA) of Protestant Support for the Nazi Party. Political Analysis, 10(3), 217-243.

O’Loughlin, J. (2003). Spatial Analysis in Political Geography. En J. Agnew, K. Mitchell y G. Toal (Eds.), A Companion to Political Geography (pp.30-46). Malden, MA: Blackwell Publishing.

O’Loughlin, J., Ward, M. D., Lofdahl, C. L., Cohen, J. S., Brown, D. S., Reilly, D.,... Shin, M. (1998). The diffusion of Democracy, 1946-1994. Annals of the Association of American Geographers, 88(4), 545-574. DOI: 10.1111/0004-5608.00112

Ord, J. K., y Getis, A. (1995). Local spatial autocorrelation statistics: distributional issues and an application. Geographical Analysis, 27(4), 286-306.

Pérez-Campuzano, E. (2011). Segregación socioespacial urbana. Debates contemporáneos e implicaciones para las ciudades mexicanas. Estudios Demográficos y Urbanos, 26(2), 403-432.

Pérez-Tamayo, B., Gil-Alonso, F., y Bayona-i-Carrasco, J. (2017). La segregación socioespacial en Culiacán, México (2000-2010): ¿de la ciudad dual a la ciudad fragmentada?. Estudios Demográficos y Urbanos, 32(3), 547-591.

Peschard, J. (1995). Cambio y continuidad en el comportamiento electoral del Distrito Federal 1988-1994. (Tesis de Doctorado en Ciencias Sociales), El Colegio de Michoacán, México.

Reardon, S. F., y O’Sullivan, D. (2004). Measures of Spatial Segregation. Sociological Methodology, 34(1), 121-162.

Riker, W. (1997). The Ferment of the 1950's and the Development of Rational Choice Theory. En K. Renwick (Ed.), Contemporary Empirical Political Theory (pp.191-201). Los Angeles, CA: University of California Press.

Rodden, J. (2010). The Geographic Distribution of Political Preference. Annual Review of Political Science, 13, 321-340.

Seabrook, N. R. (2009). The Obama Effect: Patterns of Geographic Clustering in the 2004 and 2008 Presidential Elections. The Forum, 7(2). DOI:10.2202/1540-8884.1308

Siegfried, A. (2010). Tableau politique de la France de l'Ouest sous la Troisiéme République ( $\left.2^{\mathrm{a}} \mathrm{ed}\right)$. Bruselas: Université de Bruxelles.

Soares, G. A. D., y Terron, S. L. (2008). Dois Lulas: a Geografia eleitoral da reeleicao (explorando conceitos, métodos e técnicas de análise geoespacial. Opiniao Pública, 14(2), 269-301.

Tam Cho, W. K., y Nicley, E. P. (2008). Geographic Proximity versus Institutions Evaluating Borders as Real Political Boundaries. American Politics Research, 36(6), 803-823. 
Tapia, C., y Leonardo A. G. (2016). Determinantes de la demanda clientelar en México: un análisis de la identidad del "cliente" en procesos electorales recientes. Estudios Sociológicos, XXXIV(102), 503-535.

Taylor, P.J., y Johnston, R. (1979). Geography of Elections. Harmondsworth: Penguin Books.

Tobler, W. (1970). A Computer Movie Simulating Urban Growth in the Detroit Region. Economic Geography, 46(Sup1), 234-240.

Unwin, A. (2000). Using your eyes - making statistics more visible with computers. Computational Statistics \& Data Analysis, 32(3-4), 303-312.

Vilalta, C. (2004). The Local Context and the Spatial Diffusion of Multiparty Competition in Urban Mexico, 1994-2000. Political Geography, 23(4), 403-423.

Vilalta, C. (2006). Sobre la espacialidad de los procesos electorales urbanos y una comparación entre las técnicas de regresión OLS y SAM. Estudios Demográficos y Urbanos, 21(1), 83-122.

Vilalta, C. (2007). El voto en una ciudad de empresarios ricos y empleados pobres: Marginación, segregación espacial y resultados electorales en la ciudad de México (1995-2000). Working Paper, EGAP 2007, Tecnológico de Monterrey. Recuperado de http://alejandria.ccm.itesm.mx/egap/documentos/EGAP-2007-06.pdf

Vilalta, C. (2008). ¿Se pueden predecir geográficamente los resultados electorales? Una aplicación del análisis de clústeres y outliers espaciales. Estudios Demográficos y Urbanos, 23(3), 571-613.

Ward, M. D., y Gleditsch, K. S. (2008). Spatial Regression Models. Thousand Oaks, CA: SAGE.

Weichelt, R. (2018). Un impulso para la Geografía Electoral: el uso de tecnologías geoespaciales para el análisis del voto de las comunidades hispanas en Estados Unidos. Geopolitica(s). Revista de estudios sobre espacio y poder, 9(1), 11-34. Recuperado de http://dx.doi.org/10.5209/GEOP.60705

Wong, D. (2003). Implementing spatial segregation measure in GIS. Computers, Environment and Urban Systems, 27(1), 53-70. 